\title{
Trabajo sin límites, salud insostenible: la intensificación del trabajo del conocimiento. Óscar Pérez Zapata. Madrid: Marcial Pons, 2019 - Colección Tesis Doctorales de la Federación Española de Sociología
}

\author{
Carlos Jesús Fernández Rodríguez \\ Departamento de Sociología, Universidad Autónoma de Madrid, España / Spain \\ carlos.fernandez@uam.es \\ http://orcid.org/0000-0002-2959-8195
}

Una de las iniciativas más relevantes de la Federación Española de Sociología ha sido la de impulsar algo tan esencial para las carreras académicas actuales como son las publicaciones científicas. Además del importante empuje que se ha dado a las dos revistas con las que actualmente cuenta la FES (RES y RASE), desde la FES se han promovido otras iniciativas que están comenzando a dar sus primeros frutos, esta vez en el terreno de las monografías de investigación. La colección Tesis Doctorales, editada por la FES en colaboración con Marcial Pons, es un esfuerzo específico destinado a dar un protagonismo y visibilidad a tesis doctorales de carácter innovador desarrolladas en el marco de los comités de investigación de la Federación. La presentación de esta iniciativa tuvo lugar en Valencia, en el marco del XIII Congreso Español de Sociología, y es, sin duda, una acción merecedora de aplauso y apoyo por parte de toda la comunidad sociológica, ya que permite abrir nuevos canales de publicación a trabajos desarrollados por investigadores jóvenes y no tan jóvenes que aportan nuevos enfoques y análisis a los problemas sociales contemporáneos.

Entre los comités, el pionero en promover el reconocimiento de este hito en la carrera investigadora ha sido, sin duda, el de sociología del trabajo, que desde 2016 (año del XII Congreso Español de Sociología) ya había premiado a la mejor tesis doctoral en su disciplina con la publicación de la misma. En el siguiente congreso, tres años después, se realizó la presentación en acto público de la monografía resultado de dicha tesis doctoral, que pasa a convertirse en el número 1 de una colección que, esperemos, se consolide como una referencia importante para los sociólogos de nuestro país. El libro final, titulado Trabajo sin límites, salud insostenible: la intensificación del trabajo del conocimiento, recoge una versión adaptada de la tesis doctoral de 2014 del profesor Óscar Pérez Zapata. En las siguientes páginas, se procederá a realizar una reseña de dicho trabajo.

En primer lugar, es necesario señalar que el libro se basa en una excelente tesis doctoral mucho más extensa (más de quinientas páginas) que había sido defendida en el otoño de 2014 en la Universidad Complutense de Madrid, y que obtuvo con total justicia la calificación de sobresaliente cum laude y el premio extraordinario de doctorado. Y enfatizo el "se basa" porque lo que tenemos es una versión algo más resumida de aquel trabajo, que se transforma en un manuscrito de casi trescientas páginas dividida en ocho capítulos e incluyendo dos prólogos. La investigación trataba, como se indicaba en su título, de analizar la relación entre salud y la intensificación del trabajo en la denominada economía del conocimiento. Una relación importante; quizá, de hecho, una de las más relevantes en los empleos de la nueva economía donde el exceso de trabajo (largas e intensas jornadas) tiene como resultado que el estrés y las enfermedades asociadas se hayan convertido en un elemento característico del trabajo contemporáneo. Fatiga, estrés, burnout, son síntomas de un nuevo malestar casi civilizatorio cuyo origen está en las nuevas condiciones laborales. Sectores como las finanzas, la consultoría o las nuevas tecnologías de la información se han visto sacudidos por importantes 
exigencias derivadas de la alta competitividad de sus mercados, y la clásica imagen del yuppie de los ochenta, adicto al trabajo, se ha terminado convirtiendo en un verdadero estándar de conducta hasta para los trabajadores más precarios. Ello afecta no solamente a la salud en sí de los empleados de las corporaciones (fatiga, cansancio), cada vez más exigidos, sino también a la conciliación familiar y laboral, resultando en una notable pérdida de calidad de vida. Según el autor, esta problemática de la intensificación del trabajo ha seguido empeorando con los años, acompañada además por la progresiva degradación de las condiciones laborales.

El primer capítulo del libro se ocupa de aproximar al lector al problema, valorando las consecuencias que tiene la intensificación del trabajo sobre la salud y la libertad de los individuos. El trasfondo es que ese volumen de trabajo excesivo se ha asociado, por parte de los discursos empresariales, a una muestra de compromiso del trabajador con la empresa y con su propia proyección profesional. En el discurso del management, este compromiso 0 engagement sería, además, supuestamente voluntario, un acto de libertad, con lo que no se ha problematizado a nivel organizacional. En el segundo capítulo, se trata de conectar sociológicamente esta tendencia a la intensificación del trabajo con una serie de dinámicas propias del capitalismo y la sociedad posfordista. De este modo, la aceleración e intensificación del cambio propio de la modernidad, y una cierta confusión respecto al significado de los ideales de bienestar y progreso han terminado derivando en una creciente dificultad para conciliar lo económico y lo social en un contexto de expansión de los mercados, generando múltiples contradicciones en la esfera del trabajo remunerado. La subjetividad y las emociones se han infiltrado de forma total en un terreno laboral sometido a una creciente competitividad y exigencia, provocando nuevas presiones sobre el individuo que lo conducen al estrés y la desorientación $y$, en los peores casos, al agotamiento y burnout (aunque no se trata de un fenómeno exclusivo de Ios trabajos cualificados: la creciente precarización también agrava estos problemas). Esta emergente relevancia de lo subjetivo en la esfera laboral es, evidentemente, un fenómeno característico de las organizaciones contemporáneas y su organización del trabajo, y a ellas se dedica precisamente el tercer capítulo, en el que se realiza un interesante recorrido por las formas de extracción de la fuerza de trabajo a lo largo de la historia. Tras múltiples sistemas disciplinarios, en la actualidad lo que predominan son las "culturas de empresa fuertes" como sofisticadas vías hacia la autoexplotación. La recurrente invocación al talento y a la flexibilidad ha servido para que en las empresas de la economía del conocimiento se generalicen actitudes de autocontrol y autodisciplina que permiten la emergencia de un "yo emprendedor", sin techo en sus aspiraciones y logros, y que, para cumplir con esas expectativas, se involucra hasta el límite con sus tareas. La importancia del engagement 0 compromiso es, así, fundamental en la empresa de hoy, como parte de una dinámica de sacrificio personal central en los nuevos discursos empresariales.

En el cuarto capítulo, el autor pone el foco en las prácticas concretas del trabajo, con el fin de valorar mejor ese proceso de intensificación. Apoyándose en investigaciones procedentes del campo de la sociología, la psicología social o los Critical Management Studies, Pérez Zapata analiza las exigencias que han aflorado en el terreno laboral, que provocan una creciente "carga mental" y que influyen en la creciente presencia de las emociones en el espacio de trabajo, además de una aceleración del ritmo de las tareas con consecuencias notables en el tiempo del trabajador (más horas en puesto de trabajo, picos de actividad, etc.). El fomento de la "pasión" por trabajar y la búsqueda sin límites de logros terminan generando una adicción al trabajo que se manifiesta en la imposibilidad de desconectar de él, lo que resulta en un conjunto importante de problemas asociados a la salud. Estos problemas y riesgos se describen en detalle en el quinto capítulo del libro, en el que se valora en qué medida la intensificación del trabajo hace insostenible mantener una buena salud. Así, las presiones temporales y la sobrecarga de trabajo (que en el caso del trabajo cognitivo influye de forma directa en la salud tanto mental como física) están generando un verdadero problema de salud pública derivado de la salud laboral. Hay un creciente interés por indagar en las causas sociales de estas enfermedades asociadas al ámbito del trabajo. Y es que el número creciente de fallecimientos derivados 
de causas cardiovasculares y la proliferación de cuadros de depresión y trastornos mentales tienen mucho que ver con los riesgos psicosociales asociados al trabajo. En este capítulo, Pérez Zapata presenta abundante información sobre la evolución del estrés laboral en Europa, muy vinculado a la consolidación de la economía del conocimiento pero también a otros factores, como la proliferación de modelos de gran flexibilidad laboral, desigualdades de género o el crecimiento de la precariedad, cuyo impacto sobre la identidad y la autoestima de ciertos colectivos laborales (particularmente mujeres y jóvenes) es muy notable.

A partir de aquí, Pérez Zapata pasa a presentar los resultados de la investigación empírica propiamente dicha, que consiste en una explotación de la Encuesta Nacional de Condiciones de Trabajo durante el periodo 1999-2010. A lo largo del sexto capítulo, el autor describe el trabajo con los datos, indicando los potenciales sesgos de la información recogida. En el extenso séptimo capítulo, núcleo central del libro, es donde procede a discutir los resultados empíricos. Aquí se nos presentan los perfiles tipo de aquellos afectados a nivel sanitario por el estrés (entre treinta y cinco y cincuenta años, con estudios universitarios, con tendencia a ser cada vez más jóvenes) que sufren los cambios que han tenido lugar en los trabajos cualificados (más autonomía y exigencia intelectual y emocional; peculiar organización del trabajo y apuesta por la multitarea; tecnología y procesos de innovación). La intensidad de las tareas se multiplica y así lo hacen también los riesgos para la salud. De acuerdo con los datos explotados, el compromiso o engagement al que hacen referencia las empresas está lejos de ser voluntario, sino que es más bien el resultado de una combinación de políticas de control; desde las organizaciones tampoco se proporcionan recursos adecuados para que el empleado pueda reducir dichos niveles de estrés. Además, permanece en la cultura de las organizaciones una suerte de fantasía del emprendedor que conduce al autoengaño de muchos de estos empleados, que o bien son incapaces de prestar atención a las señales del cuerpo, o caen en actitudes cercanas al cinismo y la simulación. Esta problemática pone en jaque la idea de que el trabajador se comporta como un homo economicus en la esfera organizacional, re- forzando la validez de otras miradas en las que el control organizacional o la autoalienación cobran mayor relevancia. Ello obliga a tratar de construir un mapa más amplio de la relación humana en el trabajo, en la que se produciría una compleja interacción entre imposición, voluntariedad real y aparente, cinismo, autoengaño, actitudes instrumentales y dobles vínculos. Y sobre todo, obliga a desechar la idea de libertad en el trabajo, al existir pocas oportunidades en el mercado y exigir las organizaciones una adaptación flexible y continua a sus asalariados. Al no existir libertad, aumenta la presión y ello provoca disfunciones que terminan afectando a la salud, que se convierte, en palabras de Pérez Zapata, en una "métrica política" que manifiesta problemas estructurales del mercado de trabajo y la cultura organizacional.

Finalmente, el octavo capítulo concluye con la investigación dando cuenta no solamente de las limitaciones de la misma (la falta de más datos), sino también de las implicaciones derivadas de la misma, que son fundamentalmente dos: por una parte, la necesidad de dar máxima prioridad a la prevención y promoción de la salud laboral; y por otra, la necesidad de afrontar investigaciones desde ópticas multidisciplinares para explorar la relación existente entre el engagement, el control en el trabajo y el fenómeno del burnout, con el fin de profundizar más en la investigación en salud laboral.

Trabajo sin límites, salud insostenible: la intensificación del trabajo del conocimiento es, indudablemente, un magnífico trabajo que cubre un hueco importante en la sociología del trabajo en castellano. Su originalidad es paradójica, por cuanto se trata de un tema poco explorado pese a ser un tema de enorme relevancia. La cultura del management contemporánea, con su énfasis en la flexibilidad, el compromiso y la competitividad, ha permeado de forma notable las prácticas de trabajo en las empresas que, en el caso además español, se articulan con tradiciones autoritarias en la gobernanza empresarial. Ello ha supuesto la extensión, en el paisaje laboral, de prácticas como largas jornadas de trabajo, recursos de personal escasos, presión por conseguir objetivos, estrés generalizado, etc. Es algo que forma parte de la experiencia cotidiana de la gente y por ello, de máxima relevancia sociológica. Además, pone en primera 
línea la importancia de la salud laboral, pues es indudable que conteniendo los riesgos psicosociales en el entorno de trabajo se contribuiría asimismo a aliviar la presión sobre los sistemas sanitarios. La investigación está muy bien argumentada y fundamentada, contando con un aparato teórico muy rico y multidisciplinar, y una narrativa clara y precisa. El libro nos da un auténtico baño de realidad en relación a las disfunciones de la cultura organizacional contemporánea y su impacto en nuestras vidas. En definitiva, se trata de un texto altamente recomendable que debería ser lectura obligatoria a todos aquellos interesados en la compleja relación entre trabajo y salud en las sociedades contemporáneas.

Solamente habría dos cuestiones que merecerían, quizá, una leve crítica. La primera tiene que ver con la siempre difícil transformación de una tesis doctoral en su formato original, con énfasis en lo demostrativo y estilo narrativo específico, a libro de investigación. Es indudable de que no es fácil reducir un manuscrito a la mitad de su extensión original. Sin embargo, quizá se podría haber hecho un esfuerzo adicional en dos direcciones. En primer lugar, en la necesidad de conservar parte del aparato estadístico y los datos, que en el libro final quedan reducidos significativamente; y en segundo lugar, en tratar de cohesionar el texto de forma que la extensión de los capítulos fuese algo más uniforme, algunos cuentan con muy pocas páginas y otros son bastante extensos, lo que genera una cierta perplejidad. La otra crítica, realmente menor, no tiene que ver con el esfuerzo del autor, sino con el diseño del libro que es, a juicio del que escribe estas líneas, quizá mejorable desde el punto de vista estético (sobre todo la portada). En todo caso, estas cuestiones no minimizan el hecho de que estamos ante un texto muy importante para la sociología del trabajo en España, y esperamos que contribuya a una línea de investigación consolidada en estas materias. Además, inaugura de forma espléndida la nueva colección de Tesis Doctorales de la FES, de la que podemos esperar interesantísimas publicaciones en el futuro. 